\title{
The effects of pneumoperitoneum and patient position on the perfusion index and pleth variability index during laparoscopic bariatric surgery
}

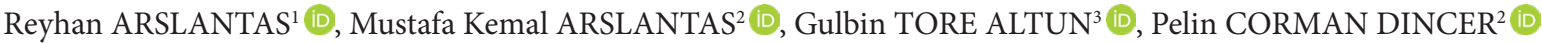 \\ Anesthesiology and Reanimation Clinic, Dr. Lutfi Kirdar Training and Research Hospital, Istanbul, Turkey. \\ Department of Anesthesiology and Reanimation, Marmara University, School of Medicine, Istanbul, Turkey. \\ Anesthesiology and Reanimation Clinic, Marmara University Pendik Training and Research Hospital, Pendik, Istanbul, Turkey.
}

Corresponding Author: Mustafa Kemal ARSLANTAS E-mail: mkarslantas@gmail.com

Submitted: $06.02 .2020 \quad$ Accepted: 26.03.2020

\section{ABSTRACT}

Objective: Limited data are available on the use of the perfusion index (PI) and the pleth variability index (PVI) during laparoscopic bariatric surgery. We investigated the effects of pneumoperitoneum and the reverse Trendelenburg position on these indices.

Materials and Methods: PI, PVI, heart rate (HR), blood pressure, $\mathrm{SpO}_{2}$ and ventilation parameters were recorded before anaesthesia induction (T0), 5 min after endotracheal intubation (T1), immediately before surgery, after the patient had been in the reverse Trendelenburg position for $5 \mathrm{~min}$ (T2), after inflating the abdomen with $\mathrm{CO}_{2}$ in reverse Trendelenburg (T3), after the abdomen had been deflated (T4) and 5 min after extubation (T5).

Results: General anaesthesia induced an increase in the PI $(\mathrm{P}<.001)$, and a decrease in the PVI $(\mathrm{P}=.002)$. The PI and PVI values were not affected during T2 or T3. Pneumoperitoneum caused an increase in mean arterial pressure (MAP) and a decrease in HR. PVI and MAP decreased during T4, but the PI and HR remained unchanged. PVI, HR and MAP increased during T5.

Conclusion: Our data suggest that the reverse Trendelenburg position and pneumoperitoneum did not affect the PI or PVI values, whereas deflation decreased the PVI in morbidly obese patients under general anaesthesia.

Keywords: Anaesthesia, Morbid obesity, Pleth variability index, Perfusion index, Bariatric surgery

\section{INTRODUCTION}

The prevalence rates of overweight and obesity are increasing worldwide, such that anaesthesiologists are encountering morbidly obese patients more frequently than before. Obesity produces a variety of hemodynamic, respiratory and metabolic changes that predispose patients to serious perioperative complications [1-4]. Hemodynamic monitoring and optimal fluid therapy are the cornerstones of therapy to improve outcomes and allow patients to tolerate the surgical procedure [5].

The perfusion index (PI) is defined as the pulsatile and nonpulsatile tissue ratio of absorbed light. The pleth variability index (PVI) is a completely non-invasive index that can be used to automatically and continuously monitor variations in the PI during the respiratory cycle, and thus has the ability to predict fluid responsiveness, and facilitate fluid management, via dynamic variables. Optimising tissue and organ perfusion through goal-directed fluid therapy based on dynamic predictors of fluid responsiveness improves clinical outcomes $[6,7]$. However, there are limitations and drawbacks to the PI and
PVI that restrict their use depending on the patient's position, pneumoperitoneum and use of norepinephrine, for example [811]. The effects of these variables have mainly been assessed in healthy individuals and non-morbidly obese patients undergoing laparoscopy [10,12]. Moreover, few studies have used the PI and PVI to predict fluid responsiveness during laparoscopic bariatric surgery [7]. To facilitate patient management and improve outcomes, anaesthetists must understand the advantages and limitations associated with the use of hemodynamic parameters to guide perioperative care in obese patients.

Therefore, we investigated the effects of pneumoperitoneum and the reverse Trendelenburg position on the PI and PVI in patients undergoing laparoscopic bariatric surgery.

\section{MATERIALS and METHODS}

The Clinical Research Ethical Committee of Marmara University, School of Medicine approved this study ( protocol no: 09.2018.737). Written informed consent was obtained from 70

How to cite this article: Arslantas R, Arslantas MK, Tore Altun G, Corman Dincer P. The effects of pneumoperitoneum and patient position on the perfusion index and pleth variability index during laparoscopic bariatric surgery. Marmara Med J 2020;33: 54-60 doi: 10.5472/marumj.711735 
patients at two hospitals, who were enrolled in this prospective observational cohort study from June 2018 to December 2019 and scheduled for elective laparoscopic bariatric surgery. The inclusion criteria were American Society of Anesthesiologists (ASA) grade II-III, age > 18 years and body mass index (BMI) $>35 \mathrm{~kg} / \mathrm{m}^{2}$.

The exclusion criteria were a history of uncompensated cardiac disease (cardiac ejection fraction $\leq 30 \%$, arrhythmias), severe respiratory disease (mechanical ventilation $\geq 8 \mathrm{ml} / \mathrm{kg}$ tidal volume) and chronic renal dysfunction.

Three patients were excluded due to conversion to open surgery, or because their PVI values could not be obtained for technical reasons, such that 67 patients were included in the final analysis.

\section{Anaesthetic procedure and monitoring}

After an overnight fast, received no premedication. Following preoxygenation, anaesthesia was induced with propofol (2$2.5 \mathrm{mg} / \mathrm{kg}$ ) based on the adjusted body weight, remifentanil (1 $\mu \mathrm{g} / \mathrm{kg}$ ) was administered based on adjusted body weight, and rocuronium $(0.6-1.2 \mathrm{mg} / \mathrm{kg})$ was administered based on total body weight; through endotracheal intubation, patients were maintained on desflurane and remifentanil according to clinical observations. Ventilation was adjusted to a tidal volume of $8-10 \mathrm{ml} / \mathrm{kg}$ based on ideal body weight, with an I:E ratio of 1:2 and positive end-expiratory pressure (PEEP) of $5 \mathrm{cmH}_{2} \mathrm{O}$. Respiration frequency and other ventilation parameters were used to guide maintenance of normocapnia $\left(\mathrm{PaCO}_{2}: 35-45\right.$ $\mathrm{mmHg}$ ) during the perioperative period. Heart rate (HR), non-invasive blood pressure, peripheral oxygen saturation, capnography, inhaled gas concentration and temperature were continuously monitored in all patients. The PI and PVI values were measured using a Masimo Radical 7 pulse oximeter probe (Masimo Radical 7; Masimo Corp., Irvine, CA, USA) attached to the right ring finger.

\section{Study protocol}

The PI and PVIvalues were obtained immediatelybefore inducing anaesthesia (T0) and after baseline hemodynamic variables were obtained. The hemodynamic and mechanical ventilation parameters were obtained $5 \mathrm{~min}$ after endotracheal intubation (T1). Immediately before surgery, the patient's position was changed to the reverse Trendelenburg position (head-up tilt to $\sim 25^{\circ}$ ), all parameters were recorded after a 5-min stabilisation period (T2), and surgery commenced. Hemodynamic and ventilation parameters were measured at 3-5 min intervals after inflating the abdomen with $\mathrm{CO}_{2}(12-15 \mathrm{mmHg}$, as measured by the insufflator) (T3), at the end of surgery when the abdomen was deflated (T4) and 5 min after extubation (T5) (Figure 1). The PI, $\mathrm{PVI}$, hemodynamic parameters $\left(\mathrm{HR}\right.$, blood pressure and $\mathrm{SpO}_{2}$ ) and ventilation parameters $\left(\mathrm{EtCO}_{2}\right.$, tidal volume, PEEP and peak airway pressure [Ppeak]) were recorded at each time point. In addition, demographic characteristics, volume of bleeding, volume of fluid administered, anaesthesia and operating times, and reverse Trendelenburg angle were recorded.

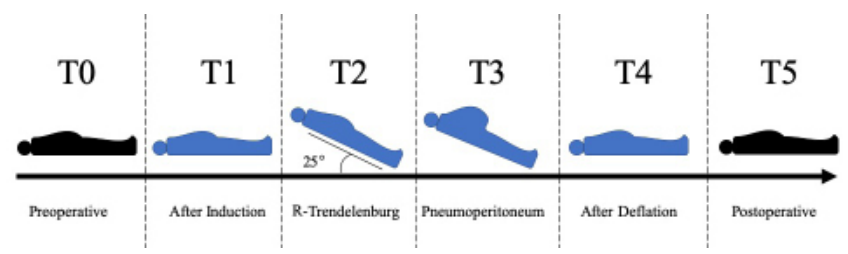

Figure 1. Illustration of the protocol of the study. T0: before endotracheal intubation; T1: $5 \mathrm{~min}$ after endotracheal intubation; T2: $5 \mathrm{~min}$ after reverse Trendelenburg; T3: 5 min after pneumoperitoneum; T4: after the termination of pneumoperitoneum; T5: $5 \mathrm{~min}$ after endotracheal extubation.

\section{Statistical analysis}

The required sample size was determined based on the data of Siswojo et al. [13]. The minimum number of samples required to determine a $2 \%$ change in the PVI values was determined to be 67 , with an alpha of 0.05 and power of 0.90 . However, we enrolled 70 patients considering the possibility of dropouts. The descriptive characteristics are expressed as frequencies and percentages for categorical variables, and as means, standard deviations and medians for numerical variables. Skewness and kurtosis values were used to assess the normality of the data. The parameters were analysed by repeated measures-analysis of variance (ANOVA). The Friedman rank-sum test was used to analyse parameters that did not meet the ANOVA assumptions. The Wilcoxon rank-sum test was used for post-hoc analysis. Because the reliability of the dynamic parameters would decrease during spontaneous breathing, comparisons of the T1-T4 measurements were made under mechanical ventilation and general anaesthesia; the T0, T1 and T5 measurements were also compared to determine the effect of general anaesthesia on the parameters. The data were analysed using $\mathrm{R}$ software (3.6.2; (R Development Core Team, Vienna, Austria). A p-value $<.05$ was considered significant.

\section{RESULTS}

Data for the demographic characteristics (age, gender, BMI, weight, height and ASA score), duration of anaesthesia and surgery, pneumoperitoneum, and angle of the reverse Trendelenburg position are presented in Table I, along with the volume of intraoperative fluid therapy (crystalloids and colloids), surgical bleeding and urine output data.

The results of the interventions are presented in Table II. General anaesthesia (T1) induced an increase in the PI $(\mathrm{P}<.001)$, and decreases in the PVI $(\mathrm{P}=.002)$ and mean arterial pressure (MAP) $(\mathrm{P}<.001)$. A decrease in MAP $(\mathrm{P}=.004)$ was seen in the reverse Trendelenburg position (T2) but the PI $(\mathrm{P}=.788)$ and PVI $(\mathrm{P}=.131)$ values were unaffected. Neither the PI nor the PVI changed significantly after pneumoperitoneum (T3) $(\mathrm{P}=.078$ and $\mathrm{P}=.397$, respectively). Neither the PI nor the PVI changed significantly after pneumoperitoneum (T3) ( $\mathrm{P}=.078$ and $\mathrm{P}=.397$, respectively). 
Table I. Distribution of the study population in terms of demographics and baseline characteristics

\begin{tabular}{lcc}
\hline & $\mathrm{n}$ & Mean \pm SD \\
\hline Age (years) & $12 / 55$ & $39.2 \pm 10.9$ \\
Gender (M/F) & & $46.7 \pm 6.0$ \\
BMI (kg/m ${ }^{2}$ & & $125.8 \pm 20.6$ \\
Weight (kg) & $163.9 \pm 8.1$ \\
Height (cm) & $44 / 23$ & $162.2 \pm 47.4$ \\
ASA score II/III & & $122.8 \pm 45.8$ \\
Duration of anaesthesia (min) & $102.1 \pm 42.6$ \\
Duration of surgery (min) & $24.5 \pm 4.3$ \\
Duration of pneumoperitoneum (min) & $1534 \pm 418$ \\
The angle of reverse Trendelenburg $\left(^{\circ}\right)$ & $450 \pm 84$ \\
Intraoperative crystalloids (ml) & 7 & $80 \pm 86$ \\
Intraoperative colloids (ml) & & $246 \pm 140$ \\
Bleeding (ml) & & \\
Urine output (ml) & \\
\hline
\end{tabular}

ASA: American Society of Anaesthesiologists physical status, BMI: body mass index, n: number of patients

Table II. Values of perfusion index (PI), pleth variability index (PVI), heart rate (HR), mean arterial blood pressure (MAP), arterial oxygen saturation measured using a pulse oximeter ( $\mathrm{SpO} 2)$, end-tidal pressure of $\mathrm{CO} 2$ (EtCO2), peak airway pressures (Ppeak), positive end-expiratory pressure (PEEP), and tidal volumes at six time points

\begin{tabular}{|c|c|c|c|c|c|c|c|}
\hline Parameters & T0 & T1 & T2 & T3 & T4 & T5 & P-value \\
\hline PI, \% & $2.12 \pm 1.58$ & $4.32 \pm 2.38$ & $4.60 \pm 2.74$ & $3.64 \pm 2.29$ & $3.24 \pm 2.52$ & $2.58 \pm 2.36$ & $<.001$ \\
\hline PVI, \% & $20.76 \pm 8.83$ & $15.76 \pm 6.65$ & $15.15 \pm 9.36$ & $14.80 \pm 6.74$ & $12.33 \pm 6.99$ & $17.70 \pm 7.12$ & $<.001$ \\
\hline HR, beats/min & $84.73 \pm 14.00$ & $85.15 \pm 13.40$ & $83.06 \pm 13.44$ & $72.91 \pm 12.99$ & $69.26 \pm 12.35$ & $92.36 \pm 16.07$ & $<.001$ \\
\hline MAP, mmHg & $97.64 \pm 14.34$ & $86.59 \pm 18.00$ & $76.62 \pm 14.22$ & $86.74 \pm 16.36$ & $78.65 \pm 11.20$ & $101.65 \pm 14.71$ & $<.001$ \\
\hline $\mathrm{SpO}_{2}, \%$ & $98.74 \pm 1.77$ & $98.77 \pm 1.54$ & $98.12 \pm 2.00$ & $97.76 \pm 1.79$ & $98.79 \pm 1.35$ & $97.60 \pm 3.32$ & $<.001$ \\
\hline $\mathrm{EtCO}_{2}, \mathrm{mmHg}$ & & $34.23 \pm 4.47$ & $32.66 \pm 3.70$ & $34.91 \pm 3.47$ & $37.97 \pm 3.13$ & & $<.001$ \\
\hline Ppeak, $\mathrm{cmH}_{2} \mathrm{O}$ & & $25.98 \pm 5.27$ & $25.02 \pm 4.61$ & $31.02 \pm 4.47$ & $27.12 \pm 4.24$ & & $<.001$ \\
\hline PEEP, $\mathrm{cmH}_{2} \mathrm{O}$ & & $6.02 \pm 1.43$ & $6.82 \pm 1.97$ & $8.06 \pm 2.05$ & $8.80 \pm 2.38$ & & $<.001$ \\
\hline Tidal Volume, ml & & $511.77 \pm 58.10$ & $519.32 \pm 52.02$ & $517.32 \pm 54.16$ & $514.60 \pm 54.25$ & & .988 \\
\hline
\end{tabular}

Data are presented as the mean \pm standard deviation. T0: before endotracheal intubation; T1: 5 min after endotracheal intubation; T2: 5 min after reverse Trendelenburg; T3: 5 min after pneumoperitoneum; T4: after the termination of pneumoperitoneum; T5: 5 min after endotracheal extubation, when appropriate

Pneumoperitoneum caused an increase in MAP $(\mathrm{P}=.001)$ and a decrease in HR $(\mathrm{P}=.013)$. The PVI $(\mathrm{P}=.028)$ and MAP $(\mathrm{P}=.007)$ decreased, whereas the PI $(\mathrm{P}=.788)$ and HR $(P=.960)$ were unchanged, after pneumoperitoneum compared to the start (T3) and termination (T4) periods. A significant decrease in the PVI was observed after release of the pneumoperitoneum (T4) compared to T1 $(\mathrm{P}=.003)$ but the PI $(\mathrm{P}=.078)$ remained unchanged (Figures 2 and 3). After extubation of the trachea (T5), the PVI $(\mathrm{P}<.001)$, HR $(\mathrm{P}<$ $.001)$ and MAP $(\mathrm{P}<.001)$ increased, but the PI $(\mathrm{P}=.078)$ did not change significantly. Significant differences were observed in the $\mathrm{SpO}_{2}$ values measured at $\mathrm{T} 3(\mathrm{P}<.001)$ and $\mathrm{T} 5(\mathrm{P}=.014)$ compared to the preoperative value (T0). $\mathrm{EtCO}_{2}$ increased gradually towards the end of the operation after inflating the abdomen with $\mathrm{CO}_{2}$. A significant difference in the EtCO2 was detected after deflation $(\mathrm{T} 4)(\mathrm{P}<.001)$ compared to the other time periods (T1-T3). Pneumoperitoneum (T3) caused an increase in Ppeak $(\mathrm{P}=.001)$ and PEEP ( $\mathrm{P}$ $=.001)$. After deflation (T4), Ppeak decreased slightly $(\mathrm{P}=$ $.090)$, but PEEP $(\mathrm{P}=.001)$ remained high, compared to the other time periods (T1 and T2). No significant difference in tidal volume was observed during the intraoperative period $(\mathrm{P}=.988)$. Mean tidal volume per ideal body weight of all patients was calculated to be $9.3 \pm 1.6 \mathrm{ml} / \mathrm{kg}$. A tidal volume $\geq 8 \mathrm{~mL} / \mathrm{kg}$ increased the reliability of the measured dynamic parameters. 


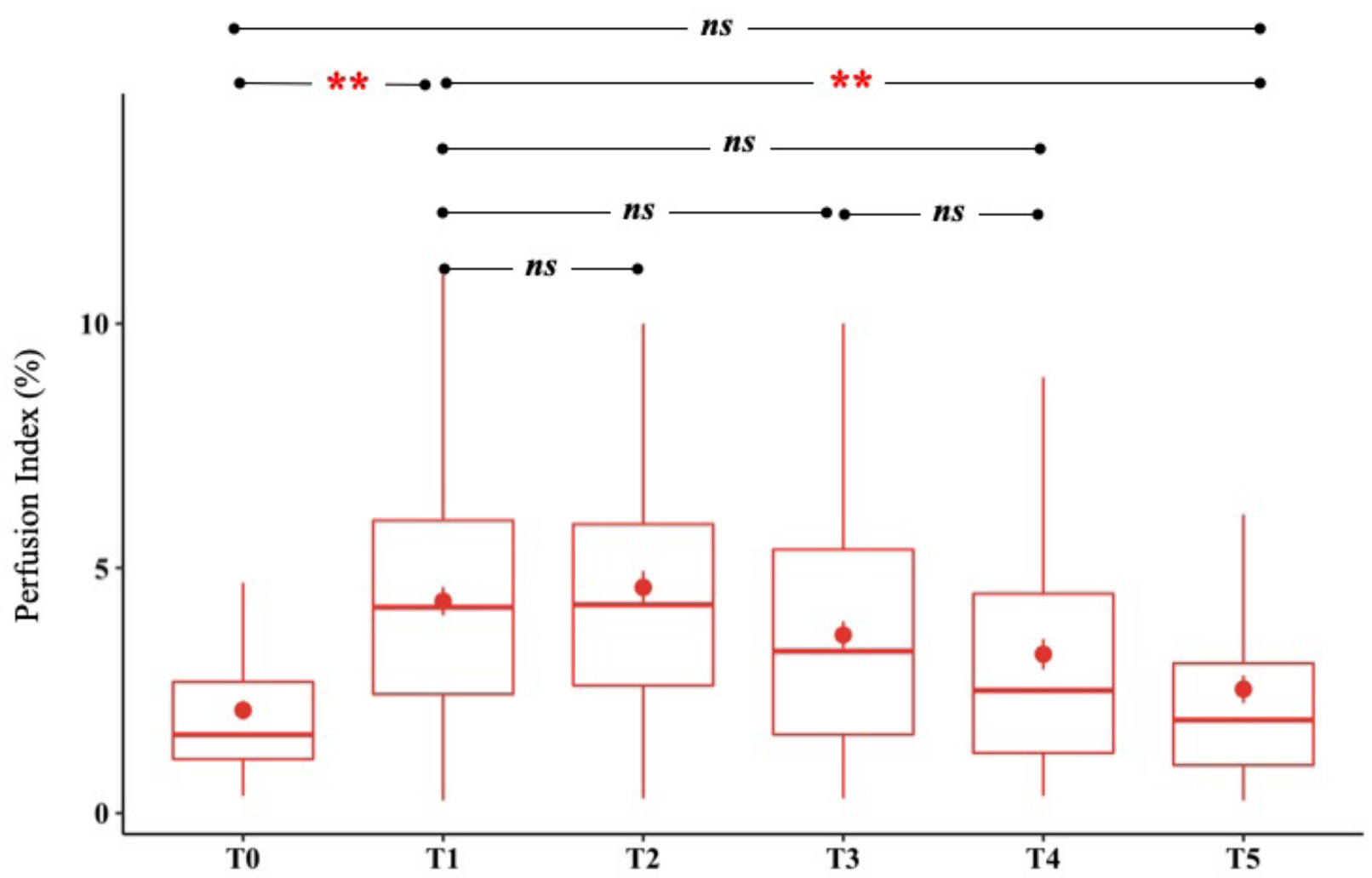

Figure 2. The values of perfusion index (PI) at six time points. ${ }^{* *} \mathrm{p}<0.001$, ns: no significant difference between time points.

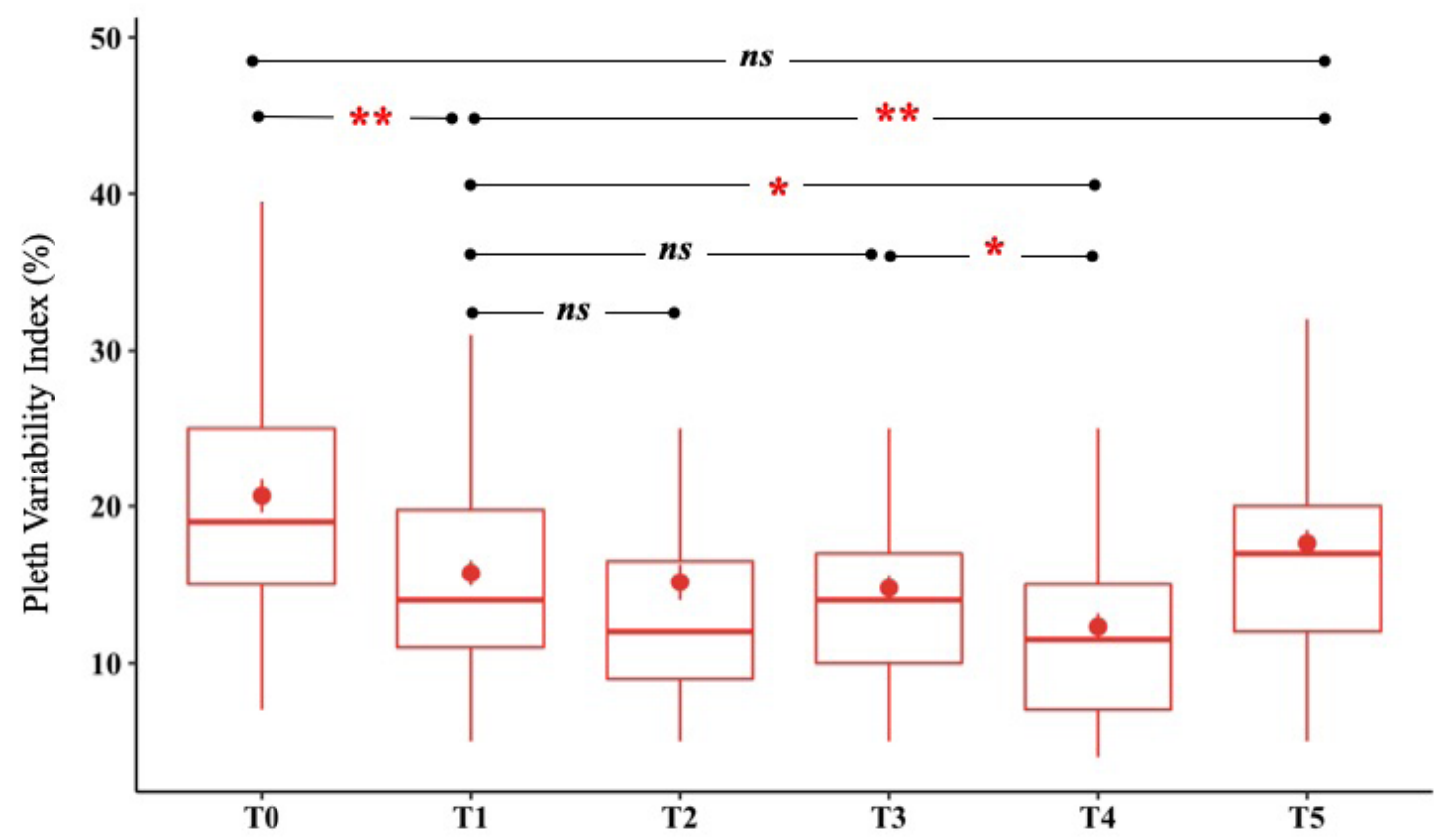

Figure 3. The values of pleth variability index (PVI) at six time points. ${ }^{\star} \mathrm{p}<0.05,{ }^{\star *} \mathrm{p}<0.001$, ns: no significant difference between time points. 


\section{DISCUSSION}

The main findings of our study are that the reverse Trendelenburg position and pneumoperitoneum did not affect the PVI or PI, whereas deflation decreased the PVI, but not the PI. In addition, the PI increased, and the PVI decreased, under general anaesthesia. Therefore, the PI and PVI can be used to guide laparoscopic bariatric surgery in patients under general anaesthesia, but not under conditions of deflation.

Our findings are consistent with an observational study, in which general anaesthesia was induced using anaesthetic agents and opioid analgesics increased the PI, but decreased the PVI [14]. These results are similar to those of our recently published article on laparoscopic cholecystectomy [9]. Administration of general anaesthetic agents and opioid analgesics causes significant changes in peripheral vascular physiology and sympathetic tone. After inducing general anaesthesia, an increase in the PI and a decrease in the PVI reflect peripheral vasodilation and decreased sympathetic tone [14]. In addition, respiratory parameters (tidal volume, ventilation rate and type [spontaneous or controlled]) also affect the PI and PVI [1416]. The decrease in PI and increase in PVI seen during the postoperative period may be related to variations in respiration during spontaneous ventilation, residual effects of intravenous or volatile anaesthetic agents and opioid analgesics, or increased sympathetic activity due to postoperative pain [16]. Therefore, we assumed that the hemodynamic parameters measured $5 \mathrm{~min}$ after endotracheal intubation (T1) could be used as baseline values when evaluating the effects of patient position and pneumoperitoneum on the PI and PVI.

The reverse Trendelenburg position can cause hemodynamic changes due to venous pooling, which can in turn lead to severe hypotension [17]. These physiological effects are similar to those associated with the seated position [17]. Bapteste et al., evaluated the utility of the PPV and PVI for predicting fluid responsiveness during general anaesthesia in the sitting position [18]. They demonstrated that, when measured with a finger sensor, the PVI was unable to predict fluid responsiveness in the sitting position. However, when measured with an ear sensor, the PVI predicted fluid responsiveness with a sensitivity of $83 \%$ and specificity of $91 \%$ [18]. We recorded the PVI using a finger sensor; our findings in the reverse Trendelenburg position might have been different if we had used an ear sensor.

Tapar et al., tested changes in the PI after patients were switched from the supine to the reverse Trendelenburg position (head-up tilt to $45^{\circ}$ ). They reported that the reverse Trendelenburg position decreased the PI significantly, which was explained by the decrease in venous return and increase in systemic vascular resistance [8]. In our hospital, the reverse Trendelenburg angle used during bariatric surgery is approximately $25^{\circ}$. In the present study, the reverse Trendelenburg position induced a decrease in MAP, but the PI and PVI values did not change; these findings were associated with the angle of the reverse Trendelenburg position and use of general anaesthesia. Tapar at al. used a head-up tilt of $45^{\circ}$ in awake participants, whereas in our patients the mean angle was $24.5 \pm 4.3^{\circ}$ under general anaesthesia; this may explain the discrepant results [8].

Intra-abdominal pressure is increased to $10-15 \mathrm{mmHg}$ by $\mathrm{CO}_{2}$ insufflation in patients undergoing laparoscopic bariatric surgery. Pneumoperitoneum can induce haemodynamic changes, which are expressed as decreases in stroke volume, cardiac output and venous return, and an increase in systemic vascular resistance [19]. Dynamic parameters, such as the PVI and stroke volume variation (SVV), may also be affected by changes in intra-abdominal pressure. Several animal and clinical studies have investigated the effects of pneumoperitoneum and/ or intra-abdominal pressure on dynamic parameters [10,2023]. These experimental studies showed that the PPV and SVV were affected by increased intra-abdominal pressure, but neither the PPV nor SVV predicted fluid responsiveness [20]. When a threshold value is used, higher than normal intra-abdominal pressure may be indicative of fluid responsiveness [21]. The results of Høiseth et al. [22] and Lui et al. [23] showed that the PVI increases and the PI decreases after pneumoperitoneum. Furthermore, the SVV increases, according to Liu et al. [23], although Høiseth et al. found that SVV was unchanged after pneumoperitoneum [22]. Wajima et al.[10] reported that pneumoperitoneum did not change the PVI, but decreased the PI and increased the SVV. These discrepancies regarding the effects of pneumoperitoneum may be related to differences in study design. For example, the anaesthesia methods (general anaesthesia or combined epidural), anaesthetic drugs (midazolam, propofol, rocuronium or cisatracurium) and opioids (remifentanil or fentanyl) used differed among studies, as did the patient characteristics (height and weight), mechanical ventilation strategies (tidal volume or PEEP). In our study, the PVI did not change after the pneumoperitoneum, similar to the results of Wajima et al. They suggested that the most likely mechanism underlying this outcome was that remifentanil and epidural analgesia block noxious stimuli and sympathetic activity in the fingertips [10]. We only administered remifentanil as an opioid to our patients; epidural anaesthesia was not used, which blocks sympathetic activity and vasoconstriction resulting from pneumoperitoneum or surgery. Høiseth et al. [12] and Lui et al. [23] used fentanyl to maintain general anaesthesia. In our study, the PI tended to decrease after pneumoperitoneum. In the study of Wajima et al. [10], the PI decreased significantly, while MAP was unchanged, after pneumoperitoneum, whereas in our study the PI tended to decrease after pneumoperitoneum, while MAP increased significantly and the HR decreased. The reason for the discrepant results is unclear, but we assume that it relates to differences in patient characteristics, and to the fact that we did not administer epidural analgesia. Unlike these previous three studies, our patients were morbidly obese; the various metabolic and neurohormonal changes commonly associated with morbid obesity may have contributed to the abnormalities in cardiac morphology and function [24].

In the present study, the PVI and MAP decreased, but the PI and $\mathrm{HR}$ were unchanged, after the pneumoperitoneum was released. We believe that this may be the result of the attenuation 
of sympathetic activity caused by increased intra-abdominal pressure.

Our study had some limitations. First, the study cohort consisted of morbidly obese patients (mean BMI $=46.7 \mathrm{~kg} / \mathrm{m}^{2}$ ), and it is well known that intra-abdominal pressure is correlated with BMI [25]. Moreover, the intra-abdominal pressure was uniformly assumed to be $0 \mathrm{mmHg}$ before pneumoperitoneum, because the actual values were unknown. However, we believe that repeated-measures design overcame this limitation. The second limitation of our study was that intra-abdominal pressure was induced by $\mathrm{CO}_{2}$ insufflation over a short period of time, which might differ from the actual conditions secondary to intra-abdominal hypertension in critical patients. Changes in the PI and PVI with increased intra-abdominal pressure must be determined in further studies.

In conclusion, our data suggest that the reverse Trendelenburg position and pneumoperitoneum did not alter the PI or PVI, whereas deflation decreased the PVI, but did not change the PI, in morbidly obese patients under general anaesthesia. Furthermore, we reconfirmed that the PI increased, while the PVI decreased, under general anaesthesia. Thus, the PI and PVI were both useful as hemodynamic parameters in patients under general anaesthesia for laparoscopic bariatric surgery, where a dose of remifentanil sufficient to block noxious stimuli was used except after release of the pneumoperitoneum.

\section{Compliance with Ethical Standards}

Funding: The authors received no financial support for the research, authorship, and/or publication of this article.

Ethics Statement: The Clinical Research Ethical Committee of Marmara University, School of Medicine approved this study ( protocol no: 09.2018.737).

\section{Conflict of Interest}

The authors declare that they have no conflict of interest.

\section{Authors' Contibutions:}

RA designed the study, carried out the experiments, gathered experimental data, performed the statistical analysis and drafted the manuscript. MKA designed the study and helped its coordination, carried out the experiments, gathered experimental data, performed the statistical analysis and revised the manuscript. GTA conceived the study, carried out the experiments, gathered experimental data, and revised the manuscript. PCD carried out the experiments, gathered experimental data and revised the manuscript. All authors read and approved the final manuscript.

\section{REFERENCES}

[1] Guh DP, Zhang W, Bansback N, Amarsi Z, Birmingham CL, Anis AH. The incidence of co-morbidities related to obesity and overweight: a systematic review and meta-analysis. BMC Public Health 2009;9:88. doi:10.1186/1471-2458-9-88
[2] Montravers P, Augustin P, Zappella N, et al. Diagnosis and management of the postoperative surgical and medical complications of bariatric surgery. Anaesth Crit Care Pain Med 2015;34:45-52. doi:10.1016/j.accpm.2014.06.002

[3] Schürner AM, Manzini G, Bueter M, Schadde E, BeckSchimmer B, Schläpfer M. Perioperative surgery - and anaesthesia-related risks of laparoscopic Roux-en-Y gastric bypass - a single centre, retrospective data analysis. BMC Anesthesiol 2018;18:190. doi:10.1186/s12871.018.0654-x

[4] Alpert MA, Omran J, Bostick BP. Effects of obesity on cardiovascular hemodynamics, cardiac morphology, and ventricular function. Curr Obes Rep 2016;5:424-34. doi:10.1007/s13679.016.0235-6

[5] Watson X, Cecconi M. Haemodynamic monitoring in the peri-operative period: the past, the present and the future. Anaesthesia 2017;72 Suppl 1:7-15. doi:10.1111/anae.13737

[6] Deng QW, Tan WC, Zhao BC, Wen SH, Shen JT, Xu M. Is goal-directed fluid therapy based on dynamic variables alone sufficient to improve clinical outcomes among patients undergoing surgery? A meta-analysis. Crit Care 2018;22:298. doi:10.1186/s13054.018.2251-2

[7] Demirel İ, Bolat E, Altun AY, Özdemir M, Beştaş A. Efficacy of goal-directed fluid therapy via pleth variability index during laparoscopic roux-en-y gastric bypass surgery in morbidly obese patients. Obes Surg 2018;28:358-63. doi:10.1007/ s11695.017.2840-1

[8] Tapar H, Karaman S, Dogru S, et al. The effect of patient positions on perfusion index. BMC Anesthesiol 2018;18:111. doi:10.1186/s12871.018.0571-Z

[9] Arslantas R, Arslantas MK, Tore Altun G, Corman Dincer P. The effect of patient position and pleth variability index in patients undergoing laparoscopic cholecystectomy. GKDA Derg 2019;25:190-7. doi: 10.5222/GKDAD.2019.80958

[10] Wajima Z, Shiga T, Imanaga K. Does pneumoperitoneum affect perfusion index and pleth variability index in patients receiving combined epidural and general anesthesia? Biosci Trends 2018;11:667-74. doi:10.5582/bst.2017.01253

[11] Monnet X, Guérin L, Jozwiak M, et al. Pleth variability index is a weak predictor of fluid responsiveness in patients receiving norepinephrine. Br J Anaesth 2013;110:207-13. doi:10.1093/ bja/aes373

[12] Høiseth LØ, Hoff IE, Hagen OA, Landsverk SA, Kirkebøen KA. Dynamic variables and fluid responsiveness in patients for aortic stenosis surgery. Acta Anaesthesiol Scand 2014;58:82634. doi:10.1111/aas.12328

[13] Siswojo AS, Wong DMY, Phan TD, Kluger R. Pleth variability index predicts fluid responsiveness in mechanically ventilated adults during general anesthesia for noncardiac surgery. J Cardiothorac Vasc Anesth [Internet]. 2014;28:1505-9. doi:10.1053/j.jvca.2014.04.010

[14] Mizuno J, Morita Y, Kakinuma A, Sawamura S. 2012. General anaesthesia induction using general anaesthetic agents and opioid analgesics increases Perfusion Index (PI) and decreases Pleth Variability Index (PVI): Observational clinical study. 
Sri Lankana J Anaesthesiol 2012; 20:7-12. doi:10.4038/slja. v20i1.3664

[15] Chu H, Wang Y, Sun Y, Wang G. Accuracy of pleth variability index to predict fluid responsiveness in mechanically ventilated patients: a systematic review and meta-analysis. J Clin Monit Comput 2016;30:265-74. doi:10.1007/s10877.015.9742-3

[16] Perel A. The value of dynamic preload variables during spontaneous ventilation. Curr Opin Crit Care 2017;23:310-7. doi:10.1097/MCC.000.000.0000000430

[17] Knight DJW, Mahajan RP. Patient positioning in anaesthesia. Contin Educ Anaesth Crit Care Pain. British Journal of Anaesthesia 2004;4:160-3. doi:10.1093/bjaceaccp/mkh044

[18] Bapteste L, Carrillon R, Javelier S, et al. Pulse pressure variations and plethysmographic variability index measured at ear are able to predict fluid responsiveness in the sitting position for neurosurgery. J Neurosurg Anesthesiol 2019;10.1097/ANA.000.000.0000000587. doi:10.1097/ ANA.000.000.0000000587

[19] Koivusalo AM, Lindgren L. Effects of carbon dioxide pneumoperitoneum for laparoscopic cholecystectomy. Acta Anaesthesiol Scand 2000;44:834-41. doi:10.1034/j.13996576.2000.440709.x

[20] Díaz F, Erranz B, Donoso A, Salomon T, Cruces P. Influence of tidal volume on pulse pressure variation and stroke volume variation during experimental intra-abdominal hypertension. BMC Anesthesiol 2015;15:127. doi:10.1186/s12871.015.0105-x

[21] Jacques D, Bendjelid K, Duperret S, Colling J, Piriou V, Viale JP. Pulse pressure variation and stroke volume variation during increased intra-abdominal pressure: an experimental study. Crit Care 2011;15:R33. doi:10.1186/cc9980

[22] Høiseth LØ, Hoff IE, Myre K, Landsverk SA, Kirkebøen KA. Dynamic variables of fluid responsiveness during pneumoperitoneum and laparoscopic surgery. Acta Anaesthesiol Scand 2012;56:777-86. doi:10.1111/j.13996576.2011.02641.x

[23] Liu F, Zhu S, Ji Q, Li W, Liu J. The impact of intra-abdominal pressure on the stroke volume variation and plethysmographic variability index in patients undergoing laparoscopic cholecystectomy. Biosci Trends. 2015;9:129-33. doi:10.5582/ bst.2015.01029

[24] Alpert MA, Omran J, Bostick BP. Effects of obesity on cardiovascular hemodynamics, cardiac morphology, and ventricular function. Curr Obes Rep 2016;5:424-34. doi:10.1007/s13679.016.0235-6

[25] Cobb WS, Burns JM, Kercher KW, Matthews BD, James Norton H, Todd Heniford B. Normal intraabdominal pressure in healthy adults. J Surg Res 2005;129:231-5. doi:10.1016/j. jss.2005.06.015 